Mitteilungen der Österreichischen Geographischen Gesellschaft, 157. Jg. (Jahresband), Wien 2015, S. 91-107

\title{
Transboundary Protected Areas als Instrument GRENZÜBERSCHREITENDER ZUSAMMENARBEIT? Der Fall des Naturparks Írottkö-Geschriebenstein
}

\author{
Martin Heintel und Norbert WeIXLBAumer, beide Wien*
}

mit 2 Abb. im Text

\section{INHALT}

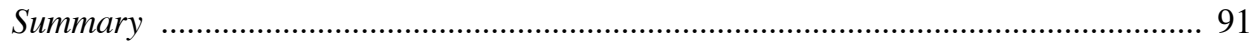

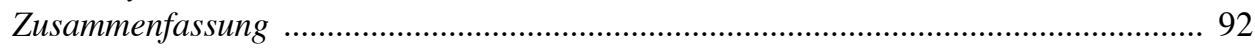

1 Grenzüberschreitende Schutzgebiete als ,Möglichkeitsräume‘ für Regional Governance Arrangements ......................................................................... 92

2 Die Entwicklung von Transboundary Protected Areas (TBPA) .............................. 93

3 Das Fallbeispiel Naturpark Írottkő-Geschriebenstein ............................................ 98

4 Regional Governance in der grenzüberschreitenden Zusammenarbeit ................. 100

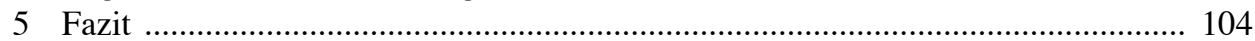

6 Literaturverzeichnis ................................................................................. 105

\section{Summary}

Transboundary Protected Areas as instruments of transborder co-operation? The case of the Nature Park Írottkö-Geschriebenstein

Transboundary Protected Areas (TBPA) represent a potential strategy for sustainable cross-border development. Experts consider them to be versatile tools for cross-border co-operation in the areas of environmental protection and peace building.

Against the backdrop of public planning of these protected areascapes, the chapter will analyse the case of a TBPA nature park located alongside the Austro-Hungarian border. Írottkö-Geschriebenstein Nature Park will serve as a model for discussing the framework conditions necessary for protected areas to play a role in cross-border co-operation at the interface of different political systems. Which mechanisms need to be considered for TBPA to act as stimuli or even models of regional development based on regional governance?

\footnotetext{
* Ao. Univ.-Prof. Mag. Dr. Martin Heintel und Ao. Univ.-Prof. Ing. Dr. Norbert Weixlbaumer, beide Institut für Geographie und Regionalforschung der Universität Wien, Universitätsstraße 7/V, 1010-Wien; E-Mail: martin. heintel@univie.ac.at, norbert.weixlbaumer@univie.ac.at
} 
Despite common ideas and statements in support of the cross-border nature park on the part of both Austria and Hungary, numerous barriers to co-operation seem to persist. From an analytical standpoint, trust, legal frameworks and jurisdictions, weak transfer links and bureaucracy, in addition to different manifestations of responsibilities as a civil society constitute those fields of action that are partly superimposed on the matters of content and need to be addressed on a deeper level in cross-border co-operation.

Keywords: bilateral co-operation, nature park, Green Belt, Irottkö-Geschriebenstein, governance arrangements

\section{Zusammenfassung}

Transboundary Protected Areas (TBPA) stellen eine mögliche Strategie für eine dauerhafte grenzüberschreitende Entwicklung dar. In Fachkreisen werden sie ausgehend von Naturschutz- und Friedensmaßnahmen als vielfältige Instrumente grenzüberschreitender Kooperationen gehandelt.

Vor dem Hintergrund der öffentlichen Planung dieser Schutzgebietskulisse wird in diesem Beitrag der Fall des TBPA Naturpark Irottkö-Geschriebenstein an der österreichisch-ungarischen Grenze analysiert. Am Beispiel dieses Naturparks wird diskutiert, unter welchen Rahmenbedingungen Schutzgebiete an der Schnittstelle von unterschiedlichen politischen Systemen ein Faktor in der grenzüberschreitenden Zusammenarbeit sein können. Welche Mechanismen sind zu berücksichtigen, damit TBPA Impulsgeber oder sogar Modell für Regional-Governance-basierte Regionalentwicklung sein können?

Es zeigt sich, dass trotz gemeinsamer Ideen und Bekenntnisse zum grenzüberschreitenden Naturpark sowohl auf österreichischer als auch auf ungarischer Seite zahlreiche Barrieren in der Zusammenarbeit bestehen. Analytisch betrachtet spiegeln Vertrauen, Rechtslage und Zuständigkeiten, Transferschwächen und Bürokratie neben unterschiedlichen Ausprägungen zivilgesellschaftlicher Verantwortungen jene Handlungsfelder wider, welche die zu gestaltenden Inhalte zum Teil überlagern und an denen in der grenzüberschreitenden Kooperation sowohl von lokalen Akteurskonstellationen als auch von institutioneller Ebene (Naturparkvereine, Verwaltungsstellen etc.) vertiefend gearbeitet werden sollte.

Schlagwörter: bilaterale Zusammenarbeit, Naturpark, Grünes Band, Írottkö-Geschriebenstein, Governance-Arrangements

\section{Grenzüberschreitende Schutzgebiete als ,Möglichkeitsräume‘ für Regional Governance Arrangements}

"We see protected areas as providers of benefits beyond boundaries - beyond their boundaries on a map, beyond the boundaries of nation states, across societies, genders and generations." (IUCN 2003)

Großschutzgebiete werden im aktuellen wissenschaftlichen Diskurs als Raumordnungsinstrumente mit mannigfachen Ansprüchen gehandelt (vgl. Mose 2007; HAMMER et 
al. 2012). Biosphärenreservate, Naturparke und Nationalparke gelten vielfach als Modellregionen zur Umsetzung von Nachhaltigkeitsinitiativen. Jede dieser Gebietskategorien (vgl. IUCN 2003) hat ihren spezifischen Eignungsbereich und kann bei Grenzgebieten hinsichtlich Raumordnung und Regionalentwicklung auch als grenzüberschreitendes Schutzgebiet Effektivität erlangen.

Diese sogenannten Transboundary Protected Areas (TBPA) (vgl. SANDwith et al. 2001; BraAcK et al. 2006; ERG, VAsiliJević \& McKinney 2012) stellen, ausgehend von Naturschutzmaßnahmen über Regionalentwicklungsprojekte bis hin zu Einstellungsfragen und Handlungsstrategien in Bezug auf Nachbarschaften ein Möglichkeitsspektrum für eine dauerhafte Entwicklung in Grenzregionen dar. Sie werden als vielversprechende und vielfältige Instrumente grenzüberschreitender Kooperationen gehandelt wie dies bei SCHOON im folgenden Zitat zum Ausdruck gebracht wird: "Transboundary protected areas have gained currency over the past decade because of their perceived (and highly disputed) effectiveness at achieving a wide array of goals ranging from improved biodiversity conservation to regional economic development to the promotion of peace between countries." (Schoon 2013, S. 421)

In der Fachliteratur wird in diesem Kontext allgemein von „Möglichkeitsräumen“ gesprochen (vgl. DAVy 2004; BeCK \& WASSENBERG 2013). Je nach Fokus und Interessen sind Grenzräume vielfach in ihrer Funktion festgelegt oder eben als sogenannte ,Möglichkeitsräume“ für die Umsetzung eines weiteren Spektrums von Interessen offen. Besonders dort, wo Nutzungen noch nicht eindeutig festgelegt oder historisch transformiert sind, wie beispielsweise in ländlichen Regionen nahe dem Eisernen Vorhang, können neue Verhältnisse im transnationalen Entwicklungsprozess geschaffen werden (vgl. BECK \& WASSENBERG 2014). HASELSBERGER (2014) spricht hier von „,soft and fuzzy spaces“ sowie von „thick borders“, die in ,thin borders“ transformiert werden können.

Die in dieser Abhandlung als Fall beleuchtete österreichisch-ungarische Grenzregion des östlichsten grenzüberschreitenden Gliedes des Alpenraumes (Írottkő-Geschriebenstein) (vgl. Abb. 1 und 2) ist so ein ,Möglichkeitsraum‘, bei welchem es im Kontext der EU-Regionalpolitik um Annäherung geht.

Als zentrale Hypothese dieses Beitrages gilt, dass Großschutzgebiete, insbesondere Naturparks, ein geeignetes Instrument sind, grenzüberschreitende Zusammenarbeit zu fördern, vor allem dann, wenn von beiden Seiten der Grenze prinzipiell positive Signale dafür gesendet werden. Die diesem Artikel zugrunde gelegten Hauptfragen lauten: Wie gestaltet sich - eingebettet in den internationalen Kontext - an diesem Fallbeispiel grenzüberschreitende Zusammenarbeit und Regionalentwicklung unter dem Policy-Prinzip von Regional Governance? Welche Voraussetzungen dafür liegen vor (Strukturfondspolitik, konzeptionelle Einbettung von grenzüberschreitenden Schutzgebieten)? Welche Interpretationsmöglichkeiten lassen die Ergebnisse des im Folgenden zu analysierenden Falls zu?

\section{Die Entwicklung von Transboundary Protected Areas (TBPA)}

Ein Blick auf die Entwicklungen von TBPA fügt sich zeitlich, thematisch und politisch in den Paradigmenwechsel der Schutzgebietspolitik (vgl. Mose \& WeIXLBAUMER 2012, 
Egner \& Jungmeier 2014) ein. Denn TBPA können besonders gegen Ende des 20. Jhs. auf eine analoge Dynamik zurückblicken. Der Weg dahin verlief allerdings langsam und regional unterschiedlich (vgl. BRAACK et al. 2006, S. 5). Historische Quellen sprechen das Aufkommen des Begriffes Peace Park dem 18. Jh. im Zusammenhang mit der friedlichen Übereinkunft zur grenzüberschreitenden Nutzung von Wald- und Fischressourcen zu (vgl. Chester 2006). Weitere Quellen bezeichnen das zwischen Schweden und Norwegen im Jahr 1914 errichtete Friedensmonument Morokulien als erstes europäisches TBPA. Das Monument wurde auf einem sechs Hektar großen Landstrich errichtet, um des hundertjährigen Friedens zwischen beiden Ländern zu gedenken. ${ }^{1)}$ Derzeit wird bilateral an einer großflächigen Ausweitung dieses Schutzgebietes gearbeitet. Die Einrichtung dieses Landstrichs als Schutzgebiet fällt zeitlich annähernd mit jener der ersten skandinavischen Nationalparks (1909) zusammen. Es war dies der Beginn der europäischen Großschutzgebiete-Politik nach US-amerikanischem Vorbild (Yellowstone 1872) und nach den Prinzipien des statisch-konservierenden Paradigmas.

Aufbauend auf diesen ersten Wurzeln einer Schutzgebietsausweisung, in der Grenzen als Scharniere betrachtet wurden, erfolgte in der Zwischenkriegszeit ein erster konkreter Schritt: In Europa und Nordamerika wurden annähernd gleichzeitig die ersten grenzüberschreitenden großräumigen Schutzgebiete etabliert.

In Europa bildete dafür das Cracow Protocol von 1924 die rechtliche Grundlage (vgl. Chester 2008; Vasilijević 2012, S. 12). Im umstrittenen Grenzgebiet von Polen und der (damaligen) Tschechoslowakei, in den Pieninen [Pieniny], wurde ein erster grenzüberschreitender Naturpark gegründet. Die Einrichtung dieses Parks verfolgte das zentrale Ziel, aus dem Ersten Weltkrieg stammende Grenzraumkonflikte beizulegen (vgl. CHESTER 2006). Es dauerte jedoch noch weitere acht Jahre, bis die TBPA zum Zwecke der ,reservation of regions for culture, wildlife, plant and local scenery protection“ auch tatsächlich eingerichtet wurde (vgl. Chester 2008, S. 1). Auch diese Ausweisung folgte, abgesehen von der Eigenschaft als Peace Park, den Intentionen des statisch-konservierenden Paradigmas. Nahezu zeitgleich erfolgte im Grenzgebiet von Kanada und den USA die Inauguration des Waterton-Glacier International Peace Parks.

Allmählich folgten weltweit weitere grenzüberschreitende Schutzgebietsausweisungen (vgl. IUCN 2003; BraAck et al. 2006; Chester 2006; Erg, Vasilijević \& McKinney 2012) - so in den 1970er Jahren in Mittelamerika der Park La Ámistad (zwischen Costa Rica und Panama), in Südostasien die Turtle Island Heritage Protected Areas (Malaysia und Philippinen) oder in Afrika der Kgalagadi Transfrontier Park an der Grenze der Republik Südafrika zu Botswana (vgl. MitTermaier et al. 2005; VAsiLiJević 2012, S. 12). Als europäisches Beispiel für diese weltumspannende Entwicklung soll an dieser Stelle noch die Ausrufung des weltweit ersten „UNESCO-MaB Trilateral Transboundary Biosphere Reserves“ in den Ostkarpaten [Východné Karpaty, Karpaty Wschodnie, Shìdnì Karpati] in Polen, der Slowakei und der Ukraine Erwähnung finden.

Die weltumspannende Entwicklung erfuhr besonders ab den 1990er Jahren eine Beschleunigung (vgl. BRAACK et al. 2006, S. 5). Im Zuge der globalen TBPA-Ausweisungen

1) Vgl. http://www.fredsmonumentet.com (06.03.2014) 
gab es verschiedene terminologische und konzeptionelle Entwicklungsstränge (vgl. ERG et al. 2012, S. 9). Hervorzuheben sind neben den TBPA die Parks for Peace, die Transboundary Conservation and Development Areas sowie die Transboundary Migratory Corridors.

$\mathrm{Zu}$ Anfang des 21. Jhs. stellte das United Nations Environement Programme-World Conservation Monitoring Centre (UNEP-WCMC) in seiner World Database on Protected Areas von 2007, Global List of TBPA, über 220 solcher konkreter Initiativen fest. Diese TBPA binden ihrerseits mehr als 3.000 individuelle Schutzgebiete ein. Eine aktuellere Untersuchung - auch über den qualitativen Stand dieser Initiativen - ist bislang noch ausständig.

TBPA werden definiert als ,,an area of land and/or sea that straddles one or more boundaries between states, sub-national units such as provinces and regions, autonomous areas and/or areas beyond the limits of national sovereignty or jurisdiction, whose constituent parts are especially dedicated to the protection and maintenance of biological diversity, and of natural and associated cultural resources, and managed cooperatively through legal or other effective means." (SANDwITH et al. 2001)

TBPA werden also grundsätzlich als Instrumente zur Konfliktbeilegung sowie als Instrumente zur Förderung der Biodiversität verstanden. Gemäß der paradigmatischen Weiterentwicklung dienen sie zudem der wirtschaftlichen (nachhaltigen) Entwicklung von Grenzräumen. Bei genauerer Betrachtung sind TBPA ein wesentlich vielschichtigeres Instrument zur grenzüberschreitenden Zusammenarbeit, das zahlreiche Möglichkeiten bietet: "The variety of purposes and contexts, both geographical and political, and the variety of methods of implementation have resulted in a fascinating array of examples of transboundary conservation in practice." (BRAACK et al. 2006, S. 3) Die Analyse der Ergebnisse einer Untersuchung des österreichisch-ungarischen Naturparks Írottkő-Geschriebenstein soll dieses Faktum transparent machen und konkretisieren (vgl. Kapitel 3 und 4).

\subsection{Prinzipien-Set zur Umsetzung von Transboundary Protected Areas}

Bei der Einrichtung von TBPA handelt es sich um die Anwendung eines komplexen Governance-Modells. Dieses beruht einerseits auf einem langfristigen Zeithorizont, andererseits auf vielschichtigen partnerschaftlichen Arrangements (FürST et al. 2006). Insgesamt ist es eine anspruchsvolle Aufgabe, die einen langen Atem und Empathie benötigt. Niewiadomsky (zit. in VasiliJević 2012, S. 16) drückt dies folgendermaßen aus: "Little will happen if there is no spirit of cooperation among the partners, no practical incentives to collaborate or understanding of potential benefits, and no intention to understand and respect each partner's different operational conditions or culture." Gemäß NIEWIADOMSKY (2006) benötigt grenzüberschreitende Zusammenarbeit einerseits ein gegenseitiges Bewusstsein für deren Mehrwert, gesetzliche und administrative Rahmenvereinbarungen, finanzielle Unterstützung sowie soziales Kapital und entsprechende Ressourcen - andererseits auch eine veränderte, ,neue Einstellung', in der sich die Bereitschaft widerspiegelt, das Grenzgebiet als eine kohärente Region sehen zu wollen. 
Die Erfahrungen aus der Praxis zeigen, dass kein einheitliches Umsetzungsmodell existiert, sondern ein Maßnehmen jedes Falls erforderlich ist (vgl. McKinNeY \& V ASILIJEVIĆ 2012, S. 24). Allerdings gibt es verschiedene verallgemeinerbare Problemlagen sowie eine auf den Erfahrungen der vergangenen Jahrzehnte beruhende Grundausrichtung, welche Maßnahmen erforderlich sind, um die Entwicklung in eine erfolgreiche Richtung zu lenken. Auf eine solche ,Road Map‘ soll im Folgenden in Anlehnung an McKinney \& VAsiliJević (2012, S. 24ff.) zusammenfassend eingegangen werden.

Das vorgeschlagene Prinzipien-Set zur grenzüberschreitenden Zusammenarbeit ist ein Set von Schlüsselkriterien, die für die Umsetzung relevant sind. Dieses Set stellt einen auf Erfahrungen beruhenden Ideenfundus dar und ist als flexibles Instrument, als ,work in progress', zu betrachten. TBPA sind demnach im Prinzip

- Katalysatoren zur grenzüberschreitenden Lösung von Fragestellungen;

- Gefäße für lokale und regionale Arrangements von Kooperationen;

- gegenseitige und grundsätzliche Vereinbarungen, Dinge willentlich angehen und Veränderungen auch visionär vornehmen zu wollen;

- Vehikel zur Ansprache gemeinsamer Interessen sowie zur Durchführung eines Interessenausgleichs;

- Plattformen für gemeinsames Lernen, Instrumente für Wissens- und Erfahrungsaustausch;

- Leitbilder unter Bedachtnahme der regionalen Situation sowie der Interessenlagen;

- Kontinuen, die Projekte und Prozesse zur Umsetzung grenzüberschreitender Schutzgebiete erfahrbar machen können;

- flexibel verortete Instrumente, welche durch Monitoring und Evaluierung des Geschehenen in ihrer Mission und ihrem Aktionsplan Korrekturen unterworfen werden können.

\subsection{Eine große Chance - vom Eisernen Vorhang zum ,Grünen Band}

Der nach dem Zweiten Weltkrieg entstandene Eiserne Vorhang hatte in Europa als politische Demarkationslinie bis 1989 Bestand. Diese gesellschaftspolitische Hürde für ein menschenwürdiges Zusammenleben zwischen Ost und West wurde für Flora und Fauna der Grenzräume zwischen Barentssee und Schwarzem Meer zur ungewollten Chance. So wurde den grenzüberschreitenden Ökosystemen für die Dauer eines knappen halben Jahrhunderts eine mehr oder weniger ungestörte Entwicklung in ihren angestammten Lebensräumen ermöglicht.

Nach dem Fall des Eisernen Vorhanges wurde von Nichtregierungs- und Regierungsorganisationen daran gearbeitet, diese Lebensräume grosso modo zu erhalten. Im Jahr 2003 wurde die Initiative Grünes Band Europa ins Leben gerufen (vgl. ZMELIK et al. 2011). Über eine Länge von ca. 12.500 Kilometern von der Barentssee an der norwegisch-russischen Grenze über die Küstenlinie der Ostsee, weiter durch Mitteleuropa hin zum Balkan und schließlich zum Schwarzen Meer erstreckt sich das Gebiet dieses Bandes. Von diesen 12.500 Kilometern verlaufen rund 1.200 Kilometer entlang der österreichischen Grenze mit insgesamt vier Nachbarländern. Das östlichste davon ist Ungarn, mit dem Österreich neben dem Nationalpark Neusiedler See/Fertő-Hanság Nemzeti Park den Naturpark Írottkő-Geschriebenstein als grenzüberschreitendes Großschutzgebiet unterhält. Beide Parks 


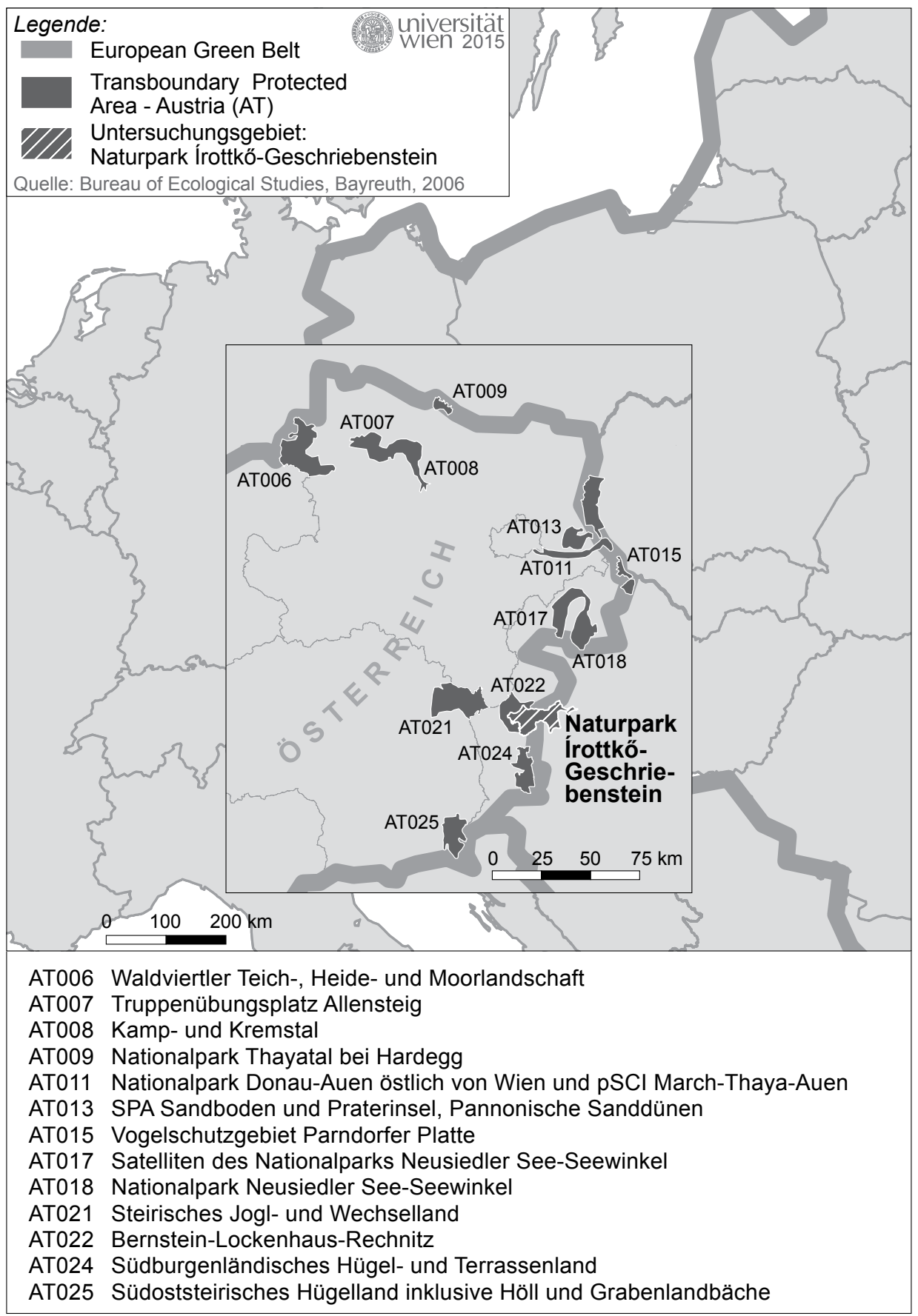

Abb. 1: Das ,Grüne Band‘ Europas und der Naturpark Írottkő-Geschriebenstein 
wurden in den 1990er Jahren eingerichtet. Dazu gesellt sich seit 1998 weiter südlich der trilaterale Naturpark Raab-Örség-Goricko.

Das ,Grüne Band‘ ist eine geopolitische Herausforderung und Chance, Naturschutzaspekte mit kulturellen Werten zu verknüpfen (vgl. GEIDEZIS \& KREUTZ 2012). Langfristiges Ziel dieser Initiative ist es, den Status eines UNESCO-Welterbes zu erreichen. Das ,Grüne Band' verbindet heute 24 Staaten und ist Rückgrat eines paneuropäischen ökologischen Netzwerkes. Darüber hinaus bildet es in sehr verschiedenen Sektoren ein Scharnier für grenzüberschreitende Zusammenarbeit. Das ,Grüne Band‘ ist ein Zeichen für den Willen zu grenzüberschreitender Zusammenarbeit und ein Bekenntnis zu einem gemeinsamen Natur- und Kulturerbe.

Offenkundig ist besonders die naturschutzfachliche Wertigkeit. Sie spiegelt sich in folgenden Fakten wider: „,40 Nationalparke [sic] liegen direkt am Grünen Band und mehr als 3.200 Schutzgebiete finden sich in einem 50 Kilometer breiten Korridor entlang des Grünen Bandes Europas. Es durchläuft fast alle europäischen biogeographischen Regionen. “" ${ }^{\text {(2) }} \mathrm{Zu}$ diesem Schutzgebietsband zählt auch der Naturpark Írottkő-Geschriebenstein, auf den im Folgenden im Kontext der Europäischen Territorialen Zusammenarbeit (ETZ) näher eingegangen wird.

\section{Das Fallbeispiel Naturpark Írottkö-Geschriebenstein}

Das ETZ-Projekt Innovativ-Grenzüberschreitend-Einheitlich-Nachhaltig (IGEN) ${ }^{3)}$ wurde von Seiten der Universität Wien in Kooperation mit den Naturparkvereinen auf österreichischer und ungarischer Seite in den Jahren 2011-2014 umgesetzt. Das Akronym IGEN bedeutet auf Ungarisch $J a$ - zu grenzüberschreitender Zusammenarbeit. Zentraler Inhalt des Projekts war es, einen Beitrag zur Organisationsentwicklung von Großschutzgebieten sowie für den Aufbau von grenzüberschreitenden Netzwerken zu leisten. Neben dem Anspruch des Projekts, bewusstseinsbildende Maßnahmen hinsichtlich der Relevanz des grenzüberschreitenden Naturparks für die ansässige Bevölkerung zu setzen, hat sich IGEN auch Ziele im Bereich der organisatorisch-institutionellen Ebene gesteckt. So soll mittels moderierter Prozessbegleitung ein grenzüberschreitendes Organisationsmodell für zukünftige Kooperationen erarbeitet werden. Das Voneinander-Lernen sowie die Identifikation der ansässigen Bevölkerung mit dem Naturpark sollen zusätzlich gefördert werden.

Da sich der grenzüberschreitende Naturpark Írottkő-Geschriebenstein an einer Schnittstelle politischer Grenzen im ,Grünen Band' befindet, ist er aufgrund seines Settings geeignet, Modellregion für eine zukunftsorientierte Organisation von TBPA im gegebenen politischen und regionalen Kontext zu sein.

Im Jahr 1995 wurden auf österreichischer Seite in Rechnitz und Lockenhaus die beiden Trägervereine für den Naturpark gegründet. Im Jahr darauf wurde der Naturpark Ge-

2) Vgl. Text aus Folder zum European Green Belt, www.europeangreenbelt.org (21.02.2014)

3) Vgl. http://www.wien.gv.at/wirtschaft/eu-strategie/eu-foerderung/etz/projekte/igen.html (29.12.2014) und http://www.at-hu.net/at-hu/de/projekte.php?we_objectID=398 (29.12.2014) 
schriebenstein offiziell durch die burgenländische Landesregierung implementiert. Bereits ein Jahr später wurde auch der ungarische Teil des Geschriebenstein-Massivs offiziell zum Naturpark erklärt. Er war damit der erste Naturpark Ungarns und zugleich der erste grenzüberschreitende Naturpark an der burgenländisch-ungarischen Grenze. Charakterisiert man ihn nach seinem Ökosystem, so ist er ein Waldnaturpark.

Das heutige Naturparkgebiet lag über mehrere Jahrzehnte an der Naht des Eisernen Vorhanges, sodass sich das Ökosystem relativ ungestört entwickeln konnte. Der Geschriebenstein (ungarisch: Irottkó) ist mit seinen 884 Höhenmetern die höchste Erhebung des Burgenlandes und zugleich der östlichste Alpengipfel. Seine Aussichtswarte - durch welche die Staatsgrenze verläuft - ist die wichtigste Landmarke des grenzüberschreitenden Naturparks.

Auf ungarischer Seite liegt mit 27.000 Hektar der größere Teil des Naturparks. Er umfasst rund um die Stadt Güns/Kőszeg das Gebiet mit den Gemeinden Bozsok, Cák, Csepreg, Kőszegdoroszló, Kőszegszerdahely und Velem. Auf österreichischer Seite erstreckt sich der Naturpark über eine Fläche von 8.500 Hektar und beinhaltet die Gemeinden Lockenhaus, Unterkohlstätten, Rechnitz und Markt Neuhodis (vgl. Abb. 2).

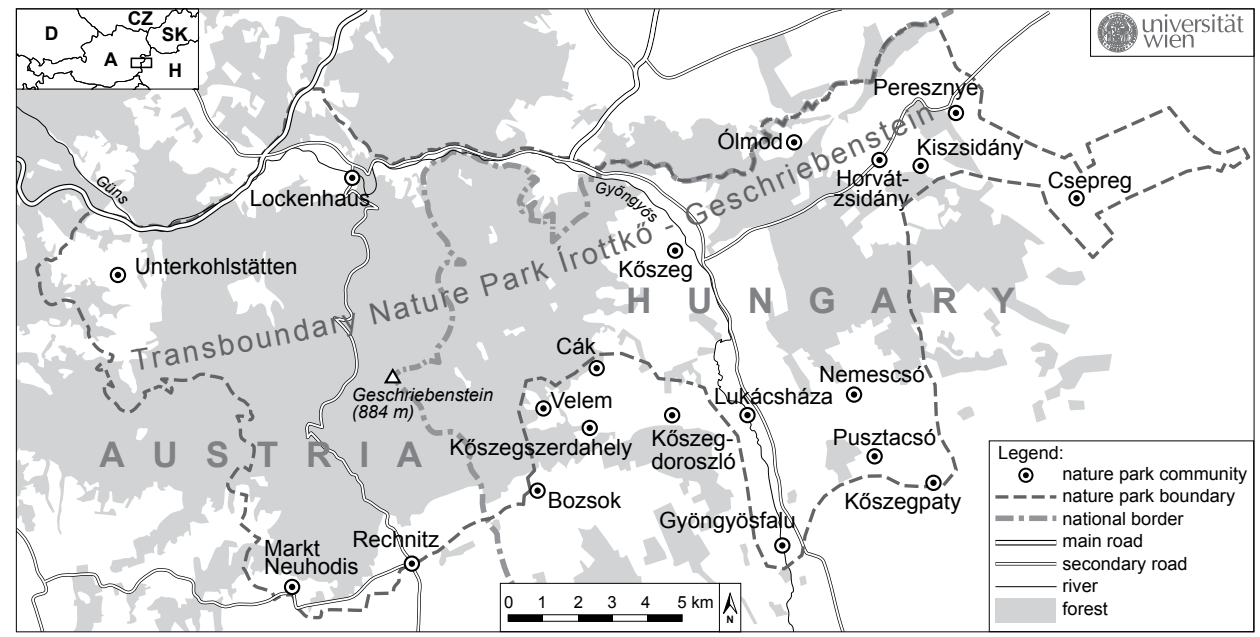

Abb. 2: Der Transboundary Nature Park Írottkő-Geschriebenstein

Ein Naturpark ist eine Prädikatslandschaft, in der auf rechtlicher Grundlage (in Österreich z.B. der eines Landschaftsschutzgebietes) nachhaltige Landschaftsentwicklung stattfinden soll. Als vom Verband der Naturparke Österreichs ausgerufenes Leitbild für diese Entwicklung dient das angestrebte „Miteinander von Schutz, Erholung, Bildung und Regionalentwicklung“ (Heintel \& Weixlbaumer 2009, S. 318). Da all diese Funktionen als gleichrangig eingestuft werden, hat ein Naturpark eine Brückenfunktion zwischen Naturschutz und Regionalentwicklung. Er fokussiert somit zugleich auf Naturschutz-, Erholungs- und Bildungsangebote und will zudem einen Beitrag zur touristischen Belebung der Region leisten. Der insgesamt 35.500 Hektar große Naturpark Írottkő-Geschrieben- 
stein bietet beispielsweise nicht nur Naturparkschulen, Informationsstellen, verschiedene Lehrpfade und einen barrierefreien Baumwipfelweg, sondern auch ein mehrere 100 Kilometer langes Wanderwegenetz.

Insgesamt existieren in Ungarn sieben staatliche Naturparks. In Österreich gibt es 48, sechs davon im Burgenland. Grundsätzlich herrschen in beiden Grenzräumen unterschiedliche rechtliche Ausgangslagen. Demgemäß wird das Naturparkkonzept auch unterschiedlich umgesetzt: In Österreich ist Naturschutz Länderkompetenz und wird durch die jeweiligen Landesnaturschutzgesetze geregelt. In Ungarn ist Naturschutz zentralistisch geregelt. So ist etwa für die Ausweisung von Schutzgebieten die nationale Ebene in Form des Ministeriums für Ländliche Entwicklung zuständig. In Ungarn werden zwar Naturparks zentralistisch ausgewiesen, Instrumente und Regelungen für eine gemeinsame Naturparkpolitik würden jedoch BASTHY (2013) zufolge fehlen. All dies macht sich in der grenzüberschreitenden Zusammenarbeit bemerkbar. So existieren kaum institutionelle Strukturen, allerdings besteht eine Reihe von informellen Kooperationsbausteinen. Das Ergebnis ist Stückwerk und lässt abgestimmte Kooperationen vermissen.

\section{Regional Governance in der grenzüberschreitenden Zusammenarbeit}

Regional Governance bezeichnet schwach institutionalisierte, mehr netzwerkartige Kooperationsformen regionaler Akteure für Aufgaben der Regionalentwicklung (vgl. u.a. Fürst 2001). Das umschreibt den bisherigen institutionellen wie informellen Handlungsrahmen der Akteure im Naturpark Írottkő-Geschriebenstein sehr gut. Auch die Österreichische Raumordnungskonferenz (ÖROK) definiert bereits in ihrem Strategischen Rahmenplan 2007 „Governance als eine horizontale Querschnittspriorität“, damit ,in einem so vielschichtigen Umfeld optimale Ergebnisse im Sinne der Regionen und Bürger erzielt werden können“. Des Weiteren wird festgehalten, dass ,alle Akteure an einem Strang ziehen müssen“. Governance wird als „das Schlagwort für diesen gemeinschaftlichen Modus“ betont (vgl. ÖROK 2007, S. 51), muss jedoch bei grenzüberschreitender Zusammenarbeit in der Regionalentwicklung zusätzliche Arrangements integrieren.

Was sind nun Kennzeichen der grenzüberschreitenden EU-Regionalpolitik im Kontext von Regional Governance?

- Vielfach kommt es zur Auslagerung von Kompetenzen und Dienstleistungen bisher hoheitlicher Planung.

- Die Abstimmung einer heterogenen Akteurslandschaft (Partnerschaftsprinzip, Partizipation, Kooperationsförderung) gewinnt dabei gleichzeitig an Komplexität.

- Netzwerkartige Arrangements sowie die Befristung von institutionellem Handeln durch die vorgegebenen Strukturfondsperioden führen letztendlich zu einer neuen Form schwacher Institutionalisierung.

- Ein Mehrebenensystem ist gefordert, die unterschiedlichen Interessen und Handlungslogiken von Bottom-up- und Top-down-Ansprüchen zusammenzuführen. 
- Das Prinzip der Kofinanzierung (z.B. Public-Private-Partnership, Zusammenführung unterschiedlicher Steuerungslogiken) und Förderabhängigkeit führt zu bürokratischen Hürden, Abhängigkeiten, zum temporären Aussetzen von Mitteln und befristeten Sicherheiten bei gleichzeitiger Unsicherheit.

\subsection{Zur Methodik und zu Ergebnissen des ETZ-Projekts}

Viele der angesprochenen Herausforderungen wurden auch während des Projekts IGEN sichtbar oder wurden von den Experten in der Region sowie im Rahmen der durchgeführten Vorarbeiten (vgl. HeInTEl \& WeIXLBAumER 2009) als solche identifiziert. Somit eignete sich der Naturpark Írottkő-Geschriebenstein als Untersuchungsgegenstand, um über vertiefende Recherchen und Analysen Handlungsmöglichkeiten und Handlungsdefizite für die grenzüberschreitende Organisation von Großschutzgebieten exemplarisch aufzuzeigen. Darüber hinaus sollte der Kooperationsprozess der beiden Naturparkvereine Írottkő-Geschriebenstein unterstützt werden.

Zu diesem Zweck wurden während der knapp dreijährigen Projektlaufzeit regelmäßige Koordinationstreffen oder Projektpartnertreffen durchgeführt - im Speziellen, um alle beteiligten Akteure einzubinden, die Vorgangsweise abzustimmen sowie Rückkoppelungen im Sinne der Interventionsforschung bereitzustellen. Auch die lokale Bevölkerung wurde von Anfang an in den Prozess einbezogen und mittels einer eigens aus dem Projekt entstandenen zweisprachigen Naturparkzeitschrift über den Fortgang des Projekts informiert.

Zur Identifikation von Potenzialen und Problemlagen wurden knapp 40 Experteninterviews als Tiefeninterviews mit Personen, deren Tätigkeit mit dem Naturpark Írottkő-Geschriebenstein zusammenhängt, auf der österreichischen und der ungarischen Seite durchgeführt. Es wurden Bürgermeister der Naturparkgemeinden, lokale Wirtschaftstreibende und Mitarbeiter von Naturschutzvereinen befragt. Inhaltlich ging es einerseits um Aspekte ihrer Arbeit und organisatorische Formen der grenzüberschreitenden Kooperation, andererseits um Verbesserungsvorschläge und touristische Vermarktung.

Es wurden Themenbereiche ausgearbeitet, auf einem Rückkoppelungsworkshop in Form von Hypothesen präsentiert und in Kleingruppen diskutiert. Anschließend ging es um die Reflexion und vertiefende Analyse der dargestellten vorläufigen Forschungsergebnisse. So wurden die unterschiedlichen Strukturen und unklaren Zuständigkeiten, welche zu mangelnder Abstimmung der Aktivitäten führen, ebenso angesprochen wie das Ungleichgewicht im Verhältnis zwischen ehrenamtlichen und hauptamtlichen Mitarbeitern und die fehlende Tradition grenzüberschreitender Kooperation.

Bei den Vorschlägen ging es um eine Verbesserung der regionalen Einbettung der Naturparkgemeinden, um die Vertiefung von Kenntnissen über Naturschutz und des Umweltbewusstseins, um eine Stärkung des gemeinsamen Marketings sowie um die Notwendigkeit einer Zukunftsstrategie mit mittel- und langfristigen Zielen. Experten und Teilnehmer sollten in einem weiteren Schritt Vorschläge zur Reduktion der Themenbereiche unterbreiten. In weiteren Bearbeitungsschritten wurden die zentralen Themenbereiche herausgefiltert, diskutiert und als verbindliche bilaterale Maßnahmen für die Zukunft proklamiert. Auch die Öffentlichkeit wurde regelmäßig über die Ergebnisse informiert. 
Die Projektergebnisse bestätigen - im Kontext der allgemeinen Problemlagen bei der Umsetzung von TBPA - die Relevanz des Prinzipien-Sets zur grenzüberschreitenden Zusammenarbeit (vgl. Kapitel 2.1). Die Erkenntnisse aus den Tiefeninterviews wurden $\mathrm{zu}$ vier Themenclustern zusammengefasst, die von den Teilnehmern in der angeführten Reihenfolge als vorrangig relevant identifiziert wurden: Kommunikation, Kooperation, Organisation und touristisches Marketing.

Als Zwischenfazit lässt sich festhalten, dass der Naturpark bei der lokalen Bevölkerung zwar bekannt zu sein scheint, jedoch nach Einschätzung der befragten Experten eher als selbstverständlicher Landschaftsraum vor der Haustür wahrgenommen wird. Sein Potenzial wird nicht erkannt. Generell identifizieren sich Bewohner in Gemeinden nahe am Geschriebenstein eher mit dem Naturpark als Bewohner entfernter gelegener Gemeinden. Zwar gibt es Freiwilligenarbeit in den österreichischen Ortsvereinen, doch die Naturparkvereine sind zu wenig im Alltagsleben der Bevölkerung präsent. Die Rolle der Naturparkschulen wird unterschätzt. Durch eine intensivere Zusammenarbeit mit diesen und den Aufbau von Naturparkschulen auch in Ungarn könnte die junge Generation für Belange des Naturparks sensibilisiert werden und damit einen Beitrag leisten, den Naturpark besser in der Region zu verankern. Der Naturpark wird zurzeit eher noch als Etikett denn als Motor für eine nachhaltige Regionalentwicklung gesehen.

Weitere Ergebnisse wurden in der sogenannten Neuhodis-Charta (benannt nach einer Naturparkgemeinde auf österreichischer Seite), die von allen teilnehmenden Verantwortlichen aus der Region unterzeichnet wurde, schriftlich festgehalten. Sie ist zweisprachig verfasst und hält folgende Punkte oder Empfehlungen für die Zukunft fest:

- Zusammenarbeit vertiefen

- zukünftig keine gemeinsame Vereinsstruktur

- zukünftig jedoch gemeinsam geregelten Kooperationsrahmen entwickeln

- Verstärkung eines gemeinsamen Auftritts bezüglich Tourismus

- Stärkung des Naturpark-Bewusstseins

- gemeinsame Produktentwicklung

Zweck der Neuhodis-Charta ist es, eine Grundlage für die weiterführende Zusammenarbeit in Händen zu haben, welche kurz und bündig festhält, worum es in der zukünftigen Kooperation zwischen den Naturpark-Partnern gehen soll. Sie stellt eine realitätsbezogene Vision im Sinne eines Leitbilds dar, an dem sich alle Beteiligten in Zukunft orientieren können und das richtungsweisend für die weitere Arbeit im Naturparkgebiet sein soll. Der gemeinsame Nenner aller Themenbereiche ist bilaterale Zusammenarbeit. Die Neuhodis-Charta stellt somit ein wichtiges Dokument für die zukünftige grenzüberschreitende Zusammenarbeit zwischen Ungarn und Österreich im Bereich des Schutzgebietsmanagements dar.

\subsection{Strukturen und Muster grenzüberschreitender Zusammenarbeit - eine Prozess- reflexion}

Analytisch gesehen stellen sich die Fragen nach den Voraussetzungen für grenzüberschreitende Zusammenarbeit im Kontext von Regional Governance hinsichtlich Legitimation, Selbstverpflichtung und gemeinsamem raumwirksamen Steuerungsanspruch. 
Welche Strukturen und Muster lassen sich am Beispiel der grenzüberschreitenden $\mathrm{Zu}$ sammenarbeit herausarbeiten? Wie etabliert sind nun Maßnahmen grenzüberschreitender Raumentwicklung am Beispiel des Projekts IGEN?

\section{Vertrauensgrundsatz}

Um im Rahmen von Governance-Arrangements und in Netzwerken zu kooperieren, bedarf es des Vertrauens gegenüber den mitwirkenden Partnern. Im Zusammenhang mit Cross-Border Regional Governance fehlt das Grundvertrauen vielfach, vor allem dann, wenn die Grenze - wie hier am Beispiel Österreich-Ungarn aufgezeigt - ehemals durch den Eisernen Vorhang bestimmt war. Die ,mentale Grenze' ist bis heute ein hemmender Faktor, wirkt generationenübergreifend und dauerhaft. Dennoch gelingt es langsam anhand von gelebter partnerschaftlicher Zusammenarbeit, wie hier am Beispiel einer TBPA, Vertrauen zu entwickeln. Vertrauen als Grundlage der Kooperation aufzubauen wird im Kontext von Cross-Border Regional Governance jedoch als sehr zäher und langsamer Prozess wahrgenommen, selbst dann, wenn es einen grundsätzlichen grenzüberschreitenden thematischen Konsens wie eine TBPA gibt. Konkurrierende Steuerungslogiken in Netzwerken (z.B. nationale Interessen versus föderale Befindlichkeiten; Wirtschaft versus Umwelt etc.) werden dann zusätzlich stärker sichtbar und führen zu Hürden im Projekt oder zu Widersprüchen.

\section{Rechtslage und Zuständigkeit}

Trotz der Möglichkeit des LEAD-Partner-Prinzips bringt bilaterale grenzüberschreitende Zusammenarbeit zumindest zwei staatliche Rechtssysteme einander näher. Zu heterogen ist die europäische Rechtslandschaft und Gesetzesvielfalt. Bei grenzüberschreitender Zusammenarbeit mit Projektpartnern an der Schnittstelle unterschiedlicher politischer Systeme wird das deutlich sichtbar. Je nach tendenziell zentralistischer (Ungarn) oder föderalistischer Ausrichtung (Österreich) des jeweiligen Mitgliedsstaats kommt dann der Institutionalisierung der Regionalentwicklung an Ort und Stelle geringere oder größere Bedeutung zu. Vielfach entstehen dadurch auch eine bilaterale Schieflage und ein Legitimationsproblem für regionales Handeln. Die ,gleiche Augenhöhe‘ der zuständigen Kooperationspartner ist dann vielfach nicht gegeben, wodurch es zu Abstimmungsproblemen und Verständnisschwierigkeiten bis hin zu persönlichen Kränkungen kommen kann. (Wer darf mit wem reden?) Vorhandene Sprachbarrieren ergänzen die Problematik.

\section{Transferschwäche und Wirksamkeit}

Good Governance als normativer Anspruch stößt bei grenzüberschreitender Zusammenarbeit oft noch schneller an Grenzen. Der in der EU-geförderten Regionalentwicklung vielfach enthaltene Auftrag, Stile der Good Governance zu übertragen, lässt sich in der grenzüberschreitenden Regionalentwicklung noch schwerer vollziehen als auf jeweils nationaler Ebene. Auch können Kooperationen, selbst wenn sie als Grundlage einer Projektbewilligung verlangt werden, nicht , verordnet ${ }^{\star}$ werden. Kooperation basiert auf Vertrauen und Freiwilligkeit und funktioniert nicht deshalb, weil sie in einem Projekt als Fördergrundlage programmatisch verankert ist. Werden Regionen als soziale Systeme betrachtet, sind die handelnden Akteure auch nicht beliebig austauschbar. Handelt es sich bei 
Grenzregionen - wie im vorliegenden Fall - auch um national periphere Lagen, wirkt in ländlich peripheren Regionen noch Strukturkonservatismus auf ein Projekt ein. Selbst am hier ausgeführten Beispiel einer TBPA, wo bilaterales Interesse zu einem Thema vorliegt, zeigen sich die ausgeführten Problemlagen.

\section{Freiwilligkeit}

Regional Governance fußt in der Regionalentwicklung auf einem hohen Grad an Freiwilligkeit. Je loser die Interaktionszusammenhänge, desto wichtiger wird Freiwilligkeit und das zivilgesellschaftliche Engagement. Das vorliegende Fallbeispiel zeigt, dass diese Grundlagen im grenzüberschreitenden Kontext mit neuen Mitgliedsstaaten vielfach fehlen. $\mathrm{Zu}$ unterschiedlich sind gesellschaftliche Voraussetzungen, Betroffenheit, Rahmenbedingungen, politisch legitimierte Verantwortungen und die einzelnen politischen Systeme als Ganze auch innerhalb der Europäischen Union. Zivilgesellschaftliches Handeln unterscheidet sich - ganz grob gesagt - zwischen den ,neuen“ Mitgliedsstaaten der EU und den , alten`EU-15. In den neuen Mitgliedsstaaten war es vielfach gewohnter Alltag, vorgegeben zu bekommen, was zu tun ist'. Die Bereitschaft, Selbstverantwortung zu übernehmen, muss somit oft erst gelernt werden, vor allem dann, wenn es um öffentliche Güter wie eine TBPA geht. Auch die Möglichkeiten, Ansprüche und Bereitschaften hinsichtlich partizipativer Planung sind somit unterschiedlich ausgeprägt.

\section{Bürokratie}

Unterschiedliche Verwaltungskulturen und -anforderungen in den einzelnen Mitgliedsstaaten steigern die Komplexität in der bilateralen Projektabwicklung. Jede der betroffenen Verwaltungsebenen will hundertprozentige Rechtssicherheit haben, das wiederum führt zu Hürden in der internen Projektkontrolle. Betroffen sind hier in Österreich z.B. die Programmverwaltung (z.B. ein Bundesland), die österreichische Prüfbehörde (Bundeskanzleramt) und die Europäische Kommission. Auch die Kofinanzierung von Projekten ist für viele Projektnehmer ein zunehmendes Problem. An der Kofinanzierung scheitern schon im Ansatz die neuen Mitgliedsstaaten. An der Gesamtlogistik scheitern immer mehr kleine Projektnehmer, die nicht die beschriebenen Ressourcen einer Großorganisation haben. In einer aktuellen Publikation der Österreichischen Raumordnungskonferenz (ÖROK) wird - wie zuvor zitiert - bereits von einem „Minderwert“ gesprochen, der durch die steigenden formalen Anforderungen dieser Programmschiene erzeugt wird (vgl. ÖROK 2013, S. 34ff.). Gleichzeitig werden ,Closed Shops' geschaffen mit einem sehr exklusiven Charakter für immer dieselben teilhabenden Akteure, die davon profitieren. Für die Zukunft wäre eine Reduktion der Bürokratie zur Abwicklung grenzüberschreitender Programme wünschenswert, was vor allem den auszuführenden Inhalten zugute käme.

\section{Fazit}

Am Beispiel des Naturparks Írottkő-Geschriebenstein an der Schnittstelle zweier konträrer politischer Systeme wurde erörtert, (a) wie sich grenzüberschreitende Zusammenarbeit oder Regionalentwicklung unter dem Prinzip von Regional Governance gestaltet und 
(b) welche Voraussetzungen notwendig sind, um grenzüberschreitende Kooperationen mittels TBPA bewerkstelligen zu können.

Werden die Analyseergebnisse aus dem Projekt IGEN zusammengefasst, so lässt sich festhalten, dass grenzüberschreitende Governance-Arrangements der Regionalentwicklung mit viel größeren formalen, mentalen und praktischen Problemen zu kämpfen haben als solche, die innerhalb eines Staates durchgeführt werden.

Trotz konsensualer Ausgangslage bleiben viele Fragen zur organisatorischen Gestaltung offen. Gemeinsame Ideen bilden zwar eine gute Grundlage für die Zusammenarbeit, sie überwinden jedoch nicht zwangsläufig die bestehenden Probleme. Auch zeigt sich, dass eine TBPA als gemeinsame Idee nicht ausreicht, um Governance-Arrangements im grenzüberschreitenden Bereich effizient wirksam werden zu lassen.

Anders als in etablierten Organisationen müssen im Bereich der Regionalentwicklung Governance-Arrangements erst geschaffen werden. Die Regeln dafür, die Verfahrensweisen und Problemlösungsmechanismen gilt es gemeinsam zu erarbeiten. Die diesbezügliche Koordinationsleistung bedeutet, Akteure aufeinander abzustimmen und gemeinsames Handeln aufzuwerten, mit dem Ziel eines Mehrwerts. Wichtig dabei ist eine gemeinsame Einschätzung der Ausgangs- und Zielsituation (vgl. Fürst 2014). Die Ergebnisse der empirischen Erhebung bestätigen zudem den allgemeinen Eindruck, dass der Aufwand für die Programmverwaltung der europäischen Strukturfondspolitik die zu gestaltenden Inhalte bei weitem überlagert und zugleich auch demotivierend auf die handelnden Akteure wirkt.

Den Tiefeninterviews zufolge bestehen in wesentlichen Bereichen von Regional-Governance-Arrangements weitere Defizite. Es sind dies unter anderem Probleme hinsichtlich - Kommunikation - beginnend bei Vertrauen, Rollenklarheit hinsichtlich der Zuständigkeiten und Bewältigungsvermögen bezüglich rechtlicher und bürokratischer Hürden;

- Organisation und Management sowie

- touristischem Marketing - allem voran im bilateralen Bereich.

Diese Defizite in den für TBPA relevantesten Kooperationsformen führen zu einem nur partiellen Erreichen der generellen Leitbildziele von Naturparks. Insgesamt spiegelt sich in diesen Problemlagen auch wider, dass das Potenzial der TBPA in der Region noch nicht ausreichend genützt werden konnte. Da die Defizite jedoch in der Zwischenzeit seitens der regionalen Akteure aufgearbeitet werden, besteht die Chance, dass mittels eines moderierten Entwicklungsprozesses das Instrument TBPA zukünftig erfolgreicher umgesetzt werden kann.

\section{Literaturverzeichnis}

BASHTY B. (2013), Ungarische Naturparke - Entwicklung durch internationale Kooperationen. In: Verband der NaturParke Österreichs (Hrsg.), Naturparke und nachhaltige Regionalentwicklung, S. 89-90. Graz.

Beck J., Wassenberg B. (Hrsg.) (2013), Grenzüberschreitende Zusammenarbeit leben und erforschen, Bd. 5: Integration und (trans-)regionale Identitäten. Stuttgart, Franz Steiner Verlag.

BeCK J., WASSENBERG B. (Hrsg.) (2014), Vivre et penser la coopération transfrontalière [Grenzüberschreitende Zusammenarbeit leben und denken], Bd. 6: Vers une cohesion territorial? [Auf 
territorialen Zusammenhalt hin?] (= Études sur l'Histoire de l'Intégration Européenne, EHIE, 26). Stuttgart, Franz Steiner Verlag.

BRAACK L. et al. (2006), Security Considerations in the Planning and Management of Transboundary Conservation Areas. Gland, IUCN.

Chester C.C. (2006), Conservation across borders: Biodiversity in an interdependent world. Washington, D.C., Islandpress.

CHESTER C. (2008), Transboundary protected areas. Retrieved from http://www.eoearth.org/view/ article/156688 (download am 03.02.2014).

Davy B. (2004), Die Neunte Stadt. Wilde Grenzen und Städteregion Ruhr 2030. Wuppertal, Müller und Busmann KG.

EGNer H., JungmeIER M. (Hrsg.) (2014), PARKS 3.0 - Protected Areas for the next Society. Klagenfurt, Verlag Johannes Heyn.

Erg B., VAsilijević M., McKinney M. (Hrsg.) (2012), Initiating effective transboundary conservation. A practitioner's guideline based on the experience from the Dinaric Arc. Gland - Belgrade, IUCN Programme Office for South-Eastern Europe.

FÜRST D. (2001), Regional governance - ein neues Paradigma der Regionalwissenschaften? In: Raumforschung und Raumordnung, 5, 6, S. 370-380.

Fürst D., Lahner M., Pollermann K. (2006), Entstehung und Funktionsweise von Regional Governance bei dem Gemeinschaftsgut Natur und Landschaft. Analysen von Place-makingund Governance-Prozessen in Biosphärenreservaten in Deutschland und Großbritannien (= Beiträge zur räumlichen Planung, 82). Hannover, Institut für Umweltplanung, Fachgruppe Landschaft, Leibnitz Universität Hannover.

FürsT D. (2014), Koordination und Führung in der Regionalplanung. In: Raumforschung und Raumordnung, 72, S. 451-462.

Geidezis L., Kreutz M. (2012), Green Belt Europe - Structure of the Initiative and Significance for a Pan European Ecological Network. In: Marschall I., Müller M., Gather M. (Hrsg.), The Green Belt as a European Ecological Network - strengths and gaps. Proceedings of the $1^{\text {st }}$ GreenNet Conference, $31^{\text {st }}$ of January 2012, Erfurt, S. 12-21. Erfurt, University of Applied Sciences Erfurt.

Hammer Th., Mose I., Scheurer Th., Siegrist D., Weixlbaumer N. (2012), Societal research perspectives on protected areas in Europe. In: eco.mont, Journal on Protected Mountain Areas Research and Management, 4, 1, S. 5-12.

HASElSBERGER B. (2014), Decoding borders. Appreciating border impacts on space and people. In: Planning Theory \& Practice, 15, 4, S. 505-526.

Heintel M., Weixlbaumer N. (2009), Die regionalökonomische Bedeutung des österreichischen Naturparktourismus. Das Beispiel Burgenland. In: Natur und Landschaft, 84, 7, S. 315 321.

InTERnATional Union for CONSERvation of Nature (IUCN) (Hrsg.) (2003), The Durban Accord. V ${ }^{\text {th }}$ IUCN World Parks Congress, Durban, South Africa, 12-13 Sept. 2003. - http://cmsdata. iucn.org/downloads/durbanaccorden.pdf (letzter Zugriff: 31.10.2015).

Mittermeier R.A. et al. (2005), Transboundary Conservation. A New Vision for Protected Areas. Mexico, Cemex.

Mose I. (Hrsg.) (2007), Protected Areas and Regional Development in Europe - Towards a New Model for the $21^{\text {st }}$ Century? London, Ashgate.

Mose I., Weixlbaumer N. (2012), A Shift of Paradigm? Protected Areas Policies in Europe in Transition - by the Example of the Hohe Tauern National Park, In: Weixlbaumer N. (Hrsg.), Anthologie zur Sozialgeographie (= Abhandlungen zur Geographie und Regionalforschung, 16), S. 106-124. Wien, Institut für Geographie und Regionalforschung. 
McKinNey M., VASILIJEvić M. (2012), Guidelines for initiating transboundary conservation. In: ERG B., Vasilijević M., McKinney M. (Hrsg.), Initiating effective transboundary conservation. A practitioner's guideline based on the experience from the Dinaric Arc, S. 24-58. Gland Belgrade, IUCN Programme Office for South-Eastern Europe.

Niewiadomski Z. (2006), Enhancing Transboundary Biodiversity Management in South Eastern Europe. Summary of the Draft Report. Vienna, UNEP.

ÖSTERREICHISCHE RAUMORDNUNGSKONFERENZ (ÖROK) (Hrsg.) (2007), STRAT.AT, Nationaler strategischer Rahmenplan Österreich (= ÖROK Schriftenreihe, 175). Wien.

ÖSTERREICHISCHE RAUMORDNUNGSKONFERENZ (ÖROK) (Hrsg.) (2013), STRAT.AT Bericht 2012 (= ÖROK Schriftenreihe, 188). Wien.

SAndwith T. et al. (2001), Transboundary Protected Areas for Peace and Co-operation. Gland Cambridge, IUCN.

Schoon M. (2013), Governance in Transboundary Conservation: How Institutional Structure and Path Dependence Matter. In: Conservation \& Society, 11, 4, pp. 420-428.

VASILIJEVIĆ M. (2012), Transboundary conservation areas: History and global trends. In: ERG B., VAsilijević M., McKinney M. (Hrsg.), Initiating effective transboundary conservation. A practitioner's guideline based on the experience from the Dinaric Arc, S. 12-16. Gland Belgrade, IUCN Programme Office for South-Eastern Europe.

ZMelik K., SCHINDLER S., WrbKa T. (2011), The European Green Belt: international collaboration in biodiversity research and nature conservation along the former Iron Curtain. Innovation. In: The European Journal of Social Science Research, 24, 3, S. 273-294. 